\title{
DEDUCCIÓN DE UN MODELO MATEMÁTICO PARA DETERMINAR LA CONCENTRACIÓN DE CONTAMINANTES EMITIDOS AL AIRE
}

\section{DEDUCTION OF A MATHEMATICAL MODEL TO DETERMINE THE CONCENTRATION OF AIR-CONTAINED CONTAMINANTS}

\author{
Valerio Bonifacio Chávez Anticona ${ }^{1}$ \\ Jorge Luis Lozano Cervera ${ }^{2}$
}

\section{RESUMEN}

El aire que respiramos en la actualidad está muy contaminado por las diferentes concentraciones de contaminantes que se emiten hacia él. Para medir el impacto que producen las concentraciones de contaminantes que se emiten al aire, existen equipos fijos y móviles que son muy costosos y en un país subdesarrollado como el nuestro, tenemos limitaciones para su adquisición, por tal motivo tenemos que buscar otra alternativa para tal fin.

Los modelos matemáticos son herramientas muy adecuadas y precisas para tratar de medir las concentraciones de los contaminantes emitidos al aire, solamente que se requiere de mucha experiencia y de mucha información para alimentar a dichos modelos.

Es por ello que en esta investigación, tratamos de deducir un modelo matemático que nos permita obtener la concentración de cualquier contaminante emitido al aire. Con la obtención de este modelo matemático, podemos predecir, hasta en el futuro, de cómo será la contaminación de cierto contaminante emitido al aire.

Palabras clave: Contaminación, aire, modelo matemático, contaminante.

\section{ABSTRACT}

AbstractThe air we breathe today is heavily contaminated by the different concentrations of pollutants emitted to it. In order to measure the impact of the concentrations of pollutants emitted to the air, there are fixed and mobile equipment that are very expensive and in an underdeveloped country like ours, we have limitations for its acquisition for that reason we have to look for another alternative for this purpose.

Mathematical models are very suitable and precise tools to try to measure the concentrations of the pollutants emitted into the air, but it requires a lot of experience and much information to feed these models.

This is why in this research we try to deduce a mathematical model that allows us to obtain the concentration of any pollutant emitted into the air. With the obtaining of this mathematical model, we can predict until the future of what will be contamination of certain pollutant emitted to the air.

Key words: Pollution, air, mathematical model, pollutant.

\section{INTRODUCCIÓN}

Trataremos describir las teorías básicas que nos permitirán deducir el modelo matemático planteado en este trabajo de investigación.

Los dos tipos existentes de fluidos, líquidos y gases, comparten las características de fluidez, lo cual permite hacer un planteamiento común a ambos, aunque existan diferencias importantes en su comportamiento. En los líquidos la distancia entre moléculas es pequeña, por lo que existe una importante fuerza de atracción especialmente visible en las fronteras que los separan de otros líquidos, gases o sólidos. Los cambios de presión debidos al movimiento apenas cambian el volumen del líquido, por lo que se les pueden considerar como fluidos incomprensibles en la mayor parte de las aplicaciones. En los gases, en cambio, la distancia intermolecular es grande y las fuerzas de interacción tan pequeñas que en un movimiento térmico aleatorio las moléculas del gas se difunden para ocupar todo el espacio disponible. También en presencia de cambios de presión relativamente pequeños se producen cambios de densidad importantes

\footnotetext{
1 Doctor en Ciencias Ambientales

Docente de la Facultad de Ciencias - Universidad Nacional Jorge Basadre Grohmann - Tacna Perú

2 Doctor en ciencias Ambientales

Docente de la Facultad de Ciencias - Universidad Nacional Jorge Basadre Grohmann - Tacna Perú
} 
(comprensibilidad). De todas formas, si las diferencias de presión debidas al movimiento son pequeñas (lo cual ocurre a velocidades bajas) y no hay efectos térmicos importantes, veremos que se puede simplificar el planteamiento del movimiento del gas suponiéndolo incomprensible. (Peña, 2000)

La masa es la propiedad que tiene un cuerpo de fluido, es la medida de la inercia o resistencia a cambiar el movimiento de éste. También es la medida de la cantidad de fluido, a la masa lo denotaremos por el símbolo $m$ (Mott \& Brito, 2006). A su vez también masa es la cantidad de materia que contiene un objeto (Ebbing et al., 2010)

La materia es el material físico del universo; es cualquier cosa que tiene masa y ocupa espacio (Brown, LeMay Jr, Bursten, \& Burdge, 2004). A su vez materia es el término general para los objetos materiales a nuestro alrededor; se le puede definir como cualquier cosa que ocupe espacio y pueda ser percibida por los sentidos (Ebbing et al., 2010)

El continuo es la materia consistente de moléculas en constante movimiento y colisión. Sin embargo, en la aproximación al continuo, se ignora la existencia de la estructura molecular y se considera una distribución continua de materia. Este punto de vista es válido si la longitud de la trayectoria libre promedio $\wedge$ de la molécula es mucho más pequeña que la dimensión de longitud I menor considerada en el problema físico. En otras palabras, el número de Knudsen (definido como $M$ ) deberá ser mucho más pequeño que la unidad, para que la hipótesis del continuo sea válida (Tirado, Sen, \& Indiana, 2009)

La convección de masa (o transferencia de masa por convección) es el mecanismo de transferencia de masa entre una superficie y un fluido en movimiento en el que intervienen tanto la difusión de masa como el movimiento de la masa de fluido. El movimiento del fluido mejora también en forma considerable la transferencia de masa, al quitar el fluido con alta concentración cercano a la superficie y reemplazarlo por el de concentración más baja que se encuentra más alejado. De modo que la razón de la convección de masa puede expresarse como:

$$
\dot{\mathrm{m}}_{\mathrm{dif}}=\mathrm{h}_{\text {masa }} \mathrm{A}_{\mathrm{s}}\left(\mathrm{C}_{\mathrm{s}}-\mathrm{C}_{\infty}\right)
$$

donde $h_{\text {masa }}$ es el coeficiente de transferencia de masa, $A_{s}$ es el área superficial y $\mathrm{C}_{\mathrm{s}}-\mathrm{C}_{\infty}$ es una diferencia apropiada de concentración de uno a otro lado de la capa límite de concentración.

La primera ley de Fick de la difusión, propuesta en 1855, afirma que la razón de difusión de una especie química en el espacio de una mezcla gaseosa (o de una solución líquida o sólida) es proporcional al gradiente de concentración de esa especie en ese lugar. Aunque una concentración más elevada para una especie significa más moléculas de ella por unidad de volumen, la concentración de una especie puede expresarse de varias maneras. A continuación, se describen dos formas comunes. (Cengel \& Ghajar, 2007)

En una base másica, la concentración se expresa en términos de densidad (o concentración de masa), la cual es la masa por unidad de volumen. $\mathrm{Si}$ se considera un volumen pequeño $\mathrm{V}$ en un lugar dentro de la mezcla, las densidades de las componentes A y B y de la mezcla A + B en ese lugar quedan dadas por

$\rho_{\mathrm{A}}=\frac{\mathrm{m}_{\mathrm{A}}}{\mathrm{v}_{\mathrm{A}+\mathrm{B}}}$

$\rho_{\mathrm{B}}=\frac{\mathrm{m}_{\mathrm{B}}}{\mathrm{v}_{\mathrm{A}+\mathrm{B}}}$

donde $V_{A+B}=V_{A}+V_{B}=V$, volumen total de la mezcla, $\mathrm{m}_{\mathrm{A}+\mathrm{B}}=\mathrm{m}_{\mathrm{A}}+\mathrm{m}_{\mathrm{B}}=\mathrm{m}$, masa total de la mezcla, $m_{A}$ y $V_{A}$, es la masa y el volumen del componente $A$ y $m_{B}$ y $V_{A}$, es la masa y el volumen del componente $\mathrm{B}$, respectivamente; a partir de las cuales se obtiene:

$$
\begin{gathered}
\rho_{\mathrm{A}}+\rho_{\mathrm{B}}=\frac{\mathrm{m}_{\mathrm{A}}}{\mathrm{V}_{\mathrm{A}+\mathrm{B}}}+\frac{\mathrm{m}_{\mathrm{B}}}{\mathrm{V}_{\mathrm{A}+\mathrm{B}}}=\frac{\mathrm{m}_{\mathrm{A}}+\mathrm{m}_{\mathrm{B}}}{\mathrm{V}_{\mathrm{A}+\mathrm{B}}}=\frac{\mathrm{m}}{\mathrm{V}} \\
=\rho
\end{gathered}
$$

resultando

$$
\rho_{\mathrm{A}}+\rho_{\mathrm{B}}=\rho \text { (1.4) }
$$

Sus unidades en el sistema internacional son $\left(\mathrm{kg} / \mathrm{m}^{3}\right)$.

Por lo tanto, la densidad de una mezcla en un lugar, es igual a la suma de las densidades de sus constituyentes en ese lugar. La concentración de masa también puede expresarse en forma adimensional en términos de la fracción de masa. Así tenemos que la fracción másica $C_{i}$, de un componente de la mezcla es el cociente entre la masa del componente y la masa total de la mezcla. Para una mezcla de A y $\mathrm{B}$, componentes tenemos:

$$
\mathrm{C}_{\mathrm{A}}=\frac{\mathrm{m}_{\mathrm{A}}}{\mathrm{m}_{\mathrm{A}+\mathrm{B}}}
$$


$\mathrm{C}_{\mathrm{A}}=\frac{\mathrm{m}_{\mathrm{A}}}{\mathrm{m}_{\mathrm{A}+\mathrm{B}}}$

En una base molar, la concentración se expresa en términos de concentración molar $\overline{\mathrm{C}}_{\mathrm{i}}$ (o densidad molar), la cual es la cantidad de materia, en kmol, por unidad de volumen. Una vez más, si se considera un volumen pequeño $\mathrm{V}$ en un lugar dentro de la mezcla, las concentraciones molares de las una componentes A y $\mathrm{B}$ y de la mezcla $\mathrm{A}+\mathrm{B}$ en ese lugar quedan dadas por

$\overline{\mathrm{C}}_{\mathrm{A}}=\frac{\rho_{\mathrm{A}}}{\mathrm{M}_{\mathrm{A}}}$

$\bar{C}_{B}=\frac{\rho_{B}}{M_{B}}$

donde $M_{A}$ y $M_{B}$, son las masas molares de las componentes $\mathrm{A}$ y $\mathrm{B}$ respectivamente, $\overline{\mathrm{C}}_{\mathrm{A}}+\overline{\mathrm{C}}_{\mathrm{B}}=$ $C$, es la concentración molar total de la mezcla. Además las masas molares de los componentes se calculan de la siguiente manera:

Masa molar de la componente $=$ Masa de la componente

$\overline{\text { Número de moles de la componente }}$

Obteniéndose $M_{A}=\frac{m_{A}}{\eta_{A}}, \quad M_{B}=\frac{m_{B}}{\eta_{B}}$; donde $\eta_{A} \quad y$ $\eta_{B}$, son el número de moles correspondientes a las componentes $\mathrm{A}$ y $\mathrm{B}$, respectivamente.

La velocidad promedio o media de la masa, $\vec{v}$, correspondiente a una mezcla de componentes múltiples se define en función de las densidades $y$ velocidades de la masa, de todas las componentes, es decir:

$\vec{v}=\frac{\sum_{\mathrm{i}} \mathrm{m}_{\mathrm{i}} \cdot \overrightarrow{\mathrm{v}}_{\mathrm{i}}}{\sum_{\mathrm{i}} \mathrm{m}_{\mathrm{i}}}=\frac{\sum_{\mathrm{i}} \mathrm{m}_{\mathrm{i}} \cdot \overrightarrow{\mathrm{v}}_{\mathrm{i}}}{\mathrm{m}}$

donde $\vec{v}_{i}$ es la velocidad absoluta de la especie i con relación a un sistema de coordenadas de referencia fijos (James, Charles, \& Robert, 1994). Para el caso de un sistema binario de las componentes A y B, tenemos:

$$
\begin{gathered}
\vec{v}=\frac{m_{A} \cdot \vec{v}_{A}+m_{B} \cdot \vec{v}_{B}}{m_{A}+m_{B}}=\frac{m_{A} \cdot \vec{v}_{A}}{m_{A}+m_{B}}+\frac{m_{B} \cdot \vec{v}_{B}}{m_{A}+m_{B}}= \\
C_{A} \cdot \vec{v}_{A}+C_{B} \cdot \vec{v}_{B}
\end{gathered}
$$

De una manera similar la velocidad molar media o promedio, $\vec{V}$, de una mezcla de componentes múltiples, se define en función de las concentraciones y velocidades molares de todas las componentes, es decir:

$\overrightarrow{\mathrm{V}}=\frac{\sum_{\mathrm{i}} \overline{\mathrm{C}}_{\mathrm{i}} \cdot \overrightarrow{\mathrm{v}}_{\mathrm{i}}}{\sum_{\mathrm{i}} \overline{\mathrm{C}}_{\mathrm{i}}}=\frac{\sum_{\mathrm{i}} \overline{\mathrm{c}}_{\mathrm{i}} \cdot \overrightarrow{\mathrm{v}}_{\mathrm{i}}}{\mathrm{C}}$
Para el caso de un sistema binario de las componentes A y B, tenemos:

$\overrightarrow{\mathrm{V}}=\frac{\overline{\mathrm{C}}_{\mathrm{A}} \cdot \overrightarrow{\mathrm{v}}_{\mathrm{A}}+\overline{\mathrm{C}}_{\mathrm{B}} \cdot \overrightarrow{\mathrm{v}}_{\mathrm{B}}}{\overline{\mathrm{C}}_{\mathrm{A}}+\overline{\mathrm{C}}_{\mathrm{B}}}=\frac{\overline{\mathrm{C}}_{\mathrm{A}} \cdot \overrightarrow{\mathrm{v}}_{\mathrm{A}}}{\mathrm{C}}+\frac{\overline{\mathrm{C}}_{\mathrm{B}} \cdot \overrightarrow{\mathrm{v}}_{\mathrm{B}}}{\mathrm{C}}=$

$\mathrm{X}_{\mathrm{A}} \cdot \overrightarrow{\mathrm{v}}_{\mathrm{A}}+\mathrm{X}_{\mathrm{B}} \cdot \overrightarrow{\mathrm{v}}_{\mathrm{B}}$ (1.13)

(James, Charles, \& Robert, 1994; Vargas \& Almera, 2008)

La ecuación de Fick de la rapidez de un flujo molar para una mezcla binaria en un sistema isotérmica e isobárica, establece lo siguiente: El flujo molar es directamente proporcional al gradiente de concentración. Se expresa matemáticamente:

Flujo molar: $\quad \overrightarrow{\mathrm{J}}_{\mathrm{Az}}^{*}=-\mathrm{C} \cdot \mathrm{D}_{\mathrm{AB}} \cdot \frac{\partial \mathrm{x}_{\mathrm{A}}}{\partial \mathrm{z}}$

$$
\text { (1.14) }
$$

Flujo másico: $\quad \overrightarrow{\mathrm{J}}_{\mathrm{Az}}=-\rho \cdot \mathrm{D}_{\mathrm{AB}} \cdot \frac{\partial \mathrm{C}_{\mathrm{A}}}{\partial \mathrm{z}}$

(1.15)

Donde:

$\mathrm{D}_{\mathrm{AB}}$ : es el coeficiente de difusión o difusividad másica del componente $\mathrm{A}$, a través del componente B. La difusividad nos indica la facilidad con que el componente $\mathrm{A}$, se transfiere en la mezcla. Si la difusividad tiene un valor elevado, entonces hay mucha facilidad para el transporte de masa. El coeficiente de difusión depende de la presión, de la temperatura y de la composición del sistema. Como es de esperar, de acuerdo con la movilidad de las moléculas, los coeficientes de difusión presentan diferencias significativas, dependiendo de la fase en que tenga lugar este mecanismo de transporte. Así, tenemos que las difusividades de los gases oscilan entre $0,5 \cdot 10^{-6}$ y $1,0 \cdot 10^{-5}$. Sus unidades en el sistema internacional son $\left(\mathrm{m}^{2} / \mathrm{s}\right)$.

$\mathrm{X}_{\mathrm{A}}$ : fracción molar del componente $\mathrm{A}$.

$\mathrm{C}_{\mathrm{A}}$ : fracción másica del componente $\mathrm{A}$

$\frac{\partial \mathrm{X}_{\mathrm{A}}}{\partial \mathrm{z}}$ : es el gradiente de concentración molar en la dirección $\mathrm{z}$.

$\frac{\partial \mathrm{C}_{\mathrm{A}}}{\partial \mathrm{z}}$ : es el gradiente de concentración másica en la dirección $\mathrm{z}$.

$\overrightarrow{\mathrm{J}}_{\mathrm{Az}}^{*}$ : es el flujo molar en la dirección de $\mathrm{z}$, relativa a la velocidad molar promedio; sus unidades en el sistema internacional son $\left(\mathrm{kmol} / \mathrm{m}^{2} . \mathrm{s}\right)$.

$\overrightarrow{\mathrm{J}}_{\mathrm{Az}}$ : es el flujo másico en la dirección de $\mathrm{z}$, relativa a la velocidad másica promedio; sus unidades en el sistema internacional son $\left(\mathrm{kg} / \mathrm{m}^{2} . \mathrm{s}\right)$.

El signo negativo indica la dirección del flujo de la concentración decreciente, es decir, de mayor a menor concentración. (Anantharaman \& Begum, 2005; Marciarelli, Stancich, \& Stappani, 2012; Vargas \& Almera, 2008) 
Teniendo en cuenta que en un volumen de control, la masa puede cruzar las fronteras $\mathrm{y}$, por consiguiente, se debe considerar la razón de la masa que entra y que sale del volumen de control (Yunus \& John, 2006), de esta manera en un VC se puede enunciar la ley de la conservación de la masa de la siguiente forma: "La transferencia neta de masa hacia o desde el volumen de control durante un intervalo de tiempo $\Delta \mathrm{t}$ es igual al cambio neto (incremento o disminución) en la masa total dentro del volumen de control durante $\Delta \mathrm{t}^{\prime \prime}$. Es decir

\{Variación neta de la masa

$\{$ dentro del VC durante $\Delta \mathrm{t}\}$

$=\left\{\begin{array}{l}\text { Masa total de la especie } \mathrm{A} \\ \text { que entra al VC durante el } \Delta \mathrm{t}\end{array}\right\}$

- $\left\{\begin{array}{l}\text { Masa total de la especie A } \\ \text { que sale del VC duarnte el } \Delta t\end{array}\right\}$

En forma simbólica tenemos

$\Delta \mathrm{m}=\mathrm{m}_{\mathrm{E}}-\mathrm{m}_{\mathrm{S}}$

donde:

$\Delta \mathrm{m}$ : es la variación de la masa dentro del volumen de control

$\mathrm{m}_{\mathrm{E}}$ : es la masa que entra al volumen de control

$\mathrm{m}_{\mathrm{S}}$ : es la masa que sale del volumen de control

(Falconi, 2011; Yunus \& John, 2006).

Teniendo en cuenta la velocidad del fluido que entra y sale del VC, se puede enunciar la ley de la conservación de la masa para la componente A, de la siguiente manera: La velocidad de acumulación de masa para la componente A dentro del VC será igual a la diferencia de la velocidad de masa de A que entra en el VC, y la velocidad de masa de $A$ que sale del volumen de control.

$\left\{\begin{array}{cccc}\text { Velocidad de acumulación } \\ \text { de } & \text { masa de } & \text { A } & \text { en el } \\ \text { VC } & \text { durante } & \text { el } & \Delta t\end{array}\right\}=$

$\left\{\begin{array}{l}\text { Velocidad de masa } \\ \text { de A que entra en } \\ \text { el VC durante el } \Delta t\end{array}\right\}-\left\{\begin{array}{l}\text { Velocidad de masa } \\ \text { de A que sale del } \\ \text { VC durante el } \Delta t\end{array}\right\}$

Para la conservación de una componente química A determinada, esta relación debe incluir un término que corresponda a la generación o desaparición de A por medio de una reacción química o biológica que ocurra dentro del volumen de control (Marciarelli, Stancich, \& Stappani, 2012).

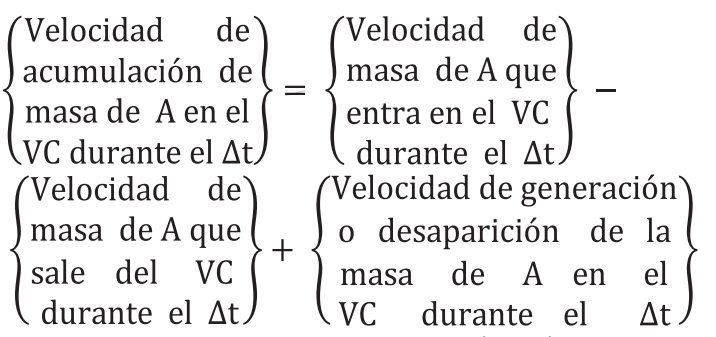

(1.18)

Por otro lado un fluido fluye hacia dentro o hacia fuera de un VC por tubos o ductos. El gasto diferencial de masa de fluido $d \vec{n}_{i}$ que fluye a través de un pequeño elemento de área, $\mathrm{dA}_{\mathrm{c}}$ (sección transversal), en una sección transversal de tubo es proporcional al propio $\mathrm{dA}_{\mathrm{c}}$, a la densidad $\rho_{\mathrm{i}}$ del fluido y a la componente de la velocidad del flujo normal a $\mathrm{dA}_{c}$, la cual lo hemos denotado como $\vec{v}_{\mathrm{i}}$, y se expresa como:

$$
d \vec{n}_{i}=\rho_{i} \cdot \vec{v}_{i} \cdot d A_{c}
$$

(Cengel \& Ghajar, 2007)

Llegándose a obtener el modelo matemático deseado para obtener la concentración de cualquier contaminante emitido al aire

$\frac{\partial \mathrm{C}_{\mathrm{A}}}{\partial \mathrm{t}}+v_{\mathrm{x}} \frac{\partial \mathrm{C}_{\mathrm{A}}}{\partial \mathrm{x}}+v_{\mathrm{y}} \frac{\partial \mathrm{C}_{\mathrm{A}}}{\partial \mathrm{y}}+v_{\mathrm{z}} \frac{\partial \mathrm{C}_{\mathrm{A}}}{\partial \mathrm{z}}-\mathrm{D}_{\mathrm{AB}}\left(\frac{\partial^{2} \mathrm{C}_{\mathrm{A}}}{\partial \mathrm{x}^{2}}+\right.$ $\left.\frac{\partial^{2} \mathrm{C}_{\mathrm{A}}}{\partial \mathrm{y}^{2}}+\frac{\partial^{2} \mathrm{C}_{\mathrm{A}}}{\partial \mathrm{z}^{2}}\right)=0$

(Boluda, 2000; Reynolds, Roth, \& Seinfeld, 1973).

\section{MATERIALES Y MÉTODO}

El área en que se enmarca esta investigación es la contaminación atmosférica la cual es impartida dentro del campo científico y en ello se desenvuelve.

En lo que concierne a la metodología que se ha seguido en esta investigación es la descriptiva, puesto que, primeramente damos a conocer en forma detallada algunas teorías básicas de la estructura de la materia y su composición, luego explicamos las unidades unitarias que participan en una mezcla, para seguidamente describir las leyes que conducen en la descripción de la dispersión de los contaminante en el aire con lo cual nos conducirán a la deducción del modelo matemático propuesto.

Para poder cumplir con la metodología planteada trataremos de revisar el material bibliográfico existente que nos permitirá entender el comportamiento de la materia en una mezcla, para así poder determinar las variables que 
participan en la deducción del modelo matemático.

En lo que a los materiales respecta se ha utilizado toda la información bibliográfica adecuada en relación a los temas que involucran a esta investigación y los accesorios correspondientes que permitirán arribar al producto final de esta investigación.

\section{RESULTADOS}

Los resultados a lo que se ha arribado en esta investigación son los siguientes:

$>\quad$ Se ha descrito las teorías básicas que nos permiten entender a la materia dentro de una mezcla; en este caso un contaminante como el soluto y el aire como el solvente.

$>\quad$ Se ha determinado los procesos que se producen dentro de una mezcla, los cuales son de importancia para la deducción del modelo matemático.

$>\quad$ Se ha determinado las leyes de la dispersión de los contaminantes, los cuales son la base para la deducción del modelo matemático.

$>\quad$ Se ha llegado a deducir el modelo matemático planteado en la presente investigación, con el cual se podrá medir la concentración de cualquier contaminante emitido al aire.

\section{DISCUSIÓN}

Existen muchos modelos matemáticos en la literatura; pero la persona que no conoce qué significa cada variable que participa en él, no podrá hacer uso de dichos modelos.

El modelo matemático que proponemos ha sido deducido paso a paso con el fin de que sea entendible por cualquier persona, que mínimamente tenga conocimientos de matemáticas y de contaminación del aire.

Creemos sin lugar a equivocarnos que el presente modelo matemático deducido será utilizado por cualquier científico que esté involucrado en estos menesteres de la contaminación del aire, y podrá compararlo con otros modelos similares y tendremos una mayor aproximación de la concentración del contaminante medido.

Los modelos matemáticos en la actualidad siempre serán de una gran importancia, si se saben aplicar correctamente, para ello tenemos que entender bien las variables que participan en él.

\section{CONCLUSIONES}

En esta investigación hemos arribado a las siguientes conclusiones:

> El modelo matemático que hemos deducido, permitirá obtener la concentración de cualquier contaminante emitido al aire.

$>$ Con este modelo matemático deducido se evitará comprar equipos que cuestan muy caro, con los cuales permiten medir las concentraciones de los contaminantes emitidos al aire.

> Con este modelo matemático se podrá predecir a futuro la concentración de un cierto contaminante emitido al aire.

> Es una primera versión de este modelo matemático el cual se podrá en el futuro seguir perfeccionando para una mejor aproximación.

\section{REFERENCIAS BIBLIOGRÁFICAS}

ANANTHARAMAN, N., \& BEGUM, K. M. M. S. (2005). Elements of mass transfer, part I. New Delhi, India: Prentice-Hall.

BROWN, T. L., LEMAY JR, H. E., BÜRSTEN, B. E., \& BURDGE, J. R. (2004). Química: La ciencia central: Prentice Hall.

CENGEL, Y. A., \& GHAJAR, A. (2007). Transferencia de calory masa. Editorial: Editorial McGraw-Hill.

Ebbing, D. D., Gammon, S. D., ZUGAZAGOITIA, J. S., CHÁVEZ, N. A. M., LANTO, J. H., \& ARRIOLA, M. A. L. (2010). Química general: Cengage Learning Editores.

MOTT, R. L., \& BRITO, J. E. (2006). Mecánica de fluidos: Pearson educación.

Peña, F. L. (2000). Mecánica de fluidos: Universidade da Coruña. Servicio de Publicacións.

TIRADO, D. A. S., SEN, M., \& INDIANA, E. (2009). Mecánica de Fluidos.

VARGAS, P., \& ALMERA, J. (2008). Fenómenos de Transporte, Fundamentos de Transporte: Recuperado de

https://ftransp.files.wordpress. com/2014/05/ transferencia-de-masa-art.pdf 